Chapter 4

\title{
Adult Mesenchymal Stem Cells in Current Tissue Engineering Concepts
}

\author{
Susanne Jung and Johannes Kleinheinz \\ Additional information is available at the end of the chapter \\ http://dx.doi.org/10.5772/59219
}

\section{Introduction}

In contemporary regenerative concepts the combination of multipotent cells and individualized scaffolds holds great promise. Adult stem cells with their regenerative and proliferative characteristics are a very adaptable cell resource for tissue regeneration.

From the support of neuronal healing processes after traumatic lesion to clinical application in plastic surgery especially adult mesenchymal stem cells have already proven their operational capability with good experimental as well as clinical success.

Human adipose derived stromal cells represent a group of mesenchymal stromal cells that come with certain advantages in terms of regenerative medicine: the cells are easily and-if necessary-repeatedly harvested, i.e. by liposuction, without leaving disfiguring scars and the cells occur in abundance, thus a relevant amount of cells can be acquired quickly. The term 'plasticity' describes the potency of adipose derived cells, to cross-differentiate across their original germ layer into cell lineages of non-mesenchymal origin. A feature that possibly opens new vistas in regenerative tissue design.

Their special charm lies not only in their differentiating ability but in their secretory properties also, that seem to be able to further support angiogenesis, inflammation and its regulation or matrix remodelling processes.

This survey aims to present the current state of adult stem cell application in regenerative concepts. With special regard to adipose-derived cells the chances and pitfalls of the appliance of these cells are illuminated. 


\section{Stem cells in regenerative medicine - Established strategies}

When talking about stem cell biology one has to define and to confirm the characteristics of this special cell population in each setting. Genuine stem cells distinguish themselves by typical biological features.

Stem cells are able to renew themselves by symmetrical division; this cell division produces two identical copies (daughter cells) from one original cell. During asymmetrical division one of the originated cells becomes the aforementioned identical daughter cell, whereas the second cell alters its cytological profile and starts irretrievably the differentiation towards a certain specialized cell type.

Figure 1 illustrates symmetrical and asymmetrical stem cell division.

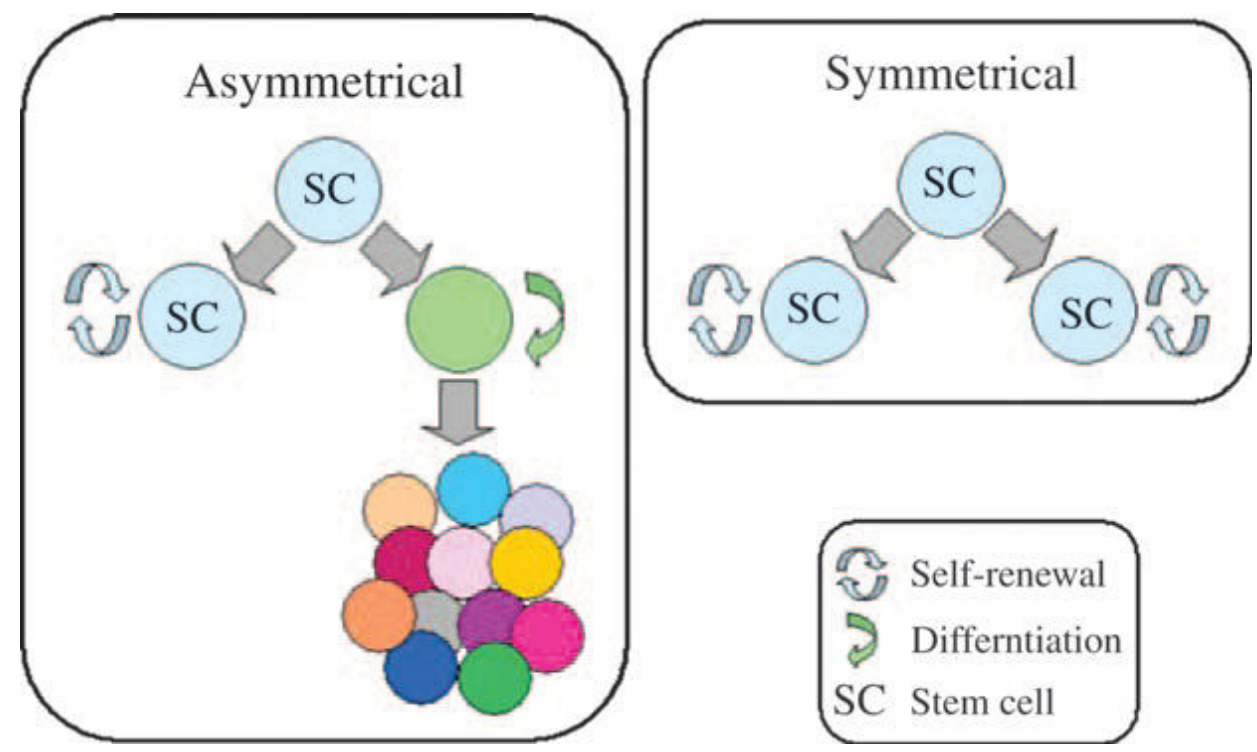

Figure 1. Symmetrical and asymmetrical stem cell division [1]

Stem cells provide a supply of progenitor cells, which are able to give rise to tissues from their original dermal layer or, especially when talking about embryonic stem cells (ESC), to tissues from all three different dermal layers. The ability to differentiate beyond the original cellular derivation means true pluripotency. In contrast, adult stem cells, harvested for example from bone marrow or the liver, are capable to generate specialized cells and tissues of their original germ layer; this ability to bring forth cells similar to their embryonic origin is called multipotency. The plasticity of adult stem cells, the potential to differentiate beyond their original germ layer in a multilineage development is discussed controversially in current literature for they have not been successful in producing veritable offspring after injection into a tetraploid blastocyst in a mouse model, so far [2]. 
A well defined panel of surface markers characterizes stem cells as multi-or pluripotent cells and separates them from other cell entities. Morphologically, for example multipotent adult cells appear as spindle-shaped, fibroblast-like cells with fine tails. In the presence of other cell populations the cells become more round, similar to cobblestones.

Yet, a morphological description alone is not able to identify the cells due to their similarity to other cells, i.e. to fibroblasts.

The immunohistochemical evidence or the typical lack of characteristic surface markers defines the cells under scrutiny as multi-or pluripotent cells. It is important to acknowledge that the expression profile of the respective cell type is dynamic and might alter during under cultivation and passaging.

Prominent defining surface markers of different human stem cells are summarized in Table 1.

\begin{tabular}{lll}
\hline ESC & MSC & ADSC \\
\hline positive & positive & positive \\
SEA-3, SSEA-4, (), TRA-1-60, TRA-1-81, CD105, CD90, CD 271, CD73 & CD13, CD29, CD44, CD49, CD90, \\
alkaline phosphatase, GTCM-2 & negative & CD105, CD106, CD166, Fibronectin, \\
negative & CD34, CD45, CD14 or CD11b, CD79a & aSMA, Vimentin, Collagen-1 \\
SSEA-1 & or CD19, MHC-II molecules, mainly & negative \\
& HLA-DR & CD 31, CD45, CD 117, CD133, HLA- \\
& & DR, STRO-1, Lin, HLA II \\
\hline
\end{tabular}

Table 1. Surface markers of different adult stem cells [3,4]

To define stem cell criteria in a cell population in short the cells in question have to show the capacity of self-renewal, they have to able to survive and proliferate long-term and the unfractioned plastic-adherent cell population has to show multipotency [5].

In many scientific constellations different groups of pluri-and mulitpotent cells have proven their effectiveness. There is a great variety of disorders in which stem cell therapy in different applications might emerge as promising therapeutic strategy.

Table 2 summarizes cell lines and corresponding diseases where stem cell therapy is under investigation.

Bai et al. investigated the effectiveness of adipose tissue derived pluripotent cells on cardiac function after acute myocardial infarction in an in vivo design and were able to show that in severe combined immunodeficient mice not only the cultivated cells but also the freshly harvested and transplanted cells showed significant positive effect on myocardial regeneration and cardiac function after local injection to the peri-infarct region [6].

Another prominent example is the application of bone marrow derived mesenchymal stem cells in the therapy of retinal degeneration. In a rat model of retinal dystrophy the subretinal or intravitreal transplantation of human bone marrow derived stem cells led to a significant amelioration of the retinal function, whereas the effect in the subretinal injection group was 
more permanent [7]. Both examples underline the potential of these cells, even if many issues remain unaddressed so far and a clinical application needs much more supportive data.

\begin{tabular}{lll}
\hline Tissue type & Cell lineage & Disease application \\
\hline Endocrine & Islet beta cells & Diabetes \\
\hline Blood & Lymphoid cells & Erythrocytes \\
\hline Liver & Hepatocyte & Sickle cell anaemia \\
\hline Renal & Podocytes and tubular cells & Liver failure \\
\hline Neural & Oligodendrocyte & Acute and chronic renal failure \\
& Dopamine neuron & Spinal cord injury \\
\hline Muscle & Motor neuron & Parkinson's disease \\
\hline Eye & Skeletal muscle & Motor neuron disease \\
\hline Cardiovascular & Retinal epithelium & Muscular dystrophy \\
& Cardiomyocyte & Retinal degeneration \\
\hline
\end{tabular}

Table 2. Tissues, cell lines and diseases

Different populations of pluripotent cells could exert their therapeutic efficacy in animal models of meniscus and lung injury as well as in clinical studies of osteogenesis imperfect [8, $9,10]$.

\section{Adult stem cells: Their pros and cons}

Stem cells derived from two different origins are investigated and applied successfully in many scientific settings and some clinical strategies: one source, the most prominent and versatile group of differentiating cells are human embryonic cells. The advantages of these cells are their ability proliferate stably over many passages in in-vitro settings and their potential to differentiate into many different cell types derived from each one of the three embryonic germ layers: the endo-, meso-and ectoderm layer [11].

This true pluripotency defines the unique attractiveness of these cells especially in regenerative concepts that aim to replace complex anatomical structures. The harvesting of embryonic cells nevertheless remains problematic and has been the reason for controversial ethical and forensic debate: human embryonic stem cells are derived from cells of the blastocyst stage of embryos; the embryos in question generally are derived from in vitro fertilization treatment and not used for implantation for different motives; to date over 1000 immortal hESC cell lines are reported. 
The process of harvesting and isolating embryonic cells is strictly regulated and under religious control. This reasonable and necessary limitation of the accessibility to the pluripotent cells complicates their use in clinical research. In addition to that, transplanted hESCs often cause strong immunorejection and as a result to their proliferative activity tend to cause neoplasia i.e. teratoma [12]. Despite the controversies, in few clinical trials dealing with spinal cord injuries, ECs are applied successfully: transplanted embryonic stem cell-derived glia cells, which are capable of producing myelin, were able to redevelop damaged spinal cord tissues in rodents with consecutive improved motoric functionality; these cells are utilised and studied in patients with complete subacute spinal cord injury to date (A Phase 1 Safety Study of GRNOPC1 in Patients With Neurologically Complete, Subacute, Spinal Cord Injury [clinical trial]. ClinicalTrials.gov registration number: NCT01217008. Accessed at http://clinicaltrials.gov/ct2/show /NCT01217008 on 11 June 2011.). The safety and tolerability of ECS derived retinal cells are investigated in patients with advanced macular degeneration, a degenerative disease that threatens the eyesight of millions of patients worldwide (A Phase I/II, Open-Label, Multi-Center, Prospective Study to Determine the Safety and Tolerability of Sub-retinal Transplantation of Human Embryonic Stem Cell Derived Retinal Pigmented Epithelial (MA09hRPE) Cells in Patients With Advanced Dry AMD [clinical trial]. ClinicalTrials.gov registration number: NCT01344993. Accessed at www.clinicaltrials.gov/ct2 /show/NCT01344993 on 11 June 2011.).

Somatic or adult stem cells, the other source of differentiating cells, can be harvested as notdifferentiated cells between surrounding specialized cells in the mature organism from various tissues or fluids without or with only little donor site morbidity. The difficulty of embryonic cell harvesting or handling does not occur.

In therapeutic concepts the use of autologous cells provides the base of personalized therapy. Their function in the embryo as well as in the adult organism is to replace and repair damaged or aged cells or tissues: this concept becomes imminent regarding the regenerative potential of the epidermis with its typical cell-based renewal cycle or the regenerative properties of the liver after damage or resection.

The advantage of these mature cells is their capacity to differentiate into various specialized cells and tissue types: mesenchymal stem cells from the bone marrow are able to develop into different cell types within the limits of their original blastodermic layer, i.e. osteoblasts or chondrocytes. Multilineage developmental or plasticity describes the stem cells' potential to overcome the histological limitations of their initial germ layer and their capacity to differentiate into progenitor cells and ultimately into cell types of another origin. This promising attribute of adult stem cells is discussed controversially in current literature:

Krampera et al. compared adult stem cells from different origins for their study: in an in vitro setting the authors were able to induce an ectodermic differentiation of MSCs from bone marrow, fat, spleen and thymus into neuron-like cells [13]. Ning et al. described in 2009 an in vivo endothelial differentiation of adipose tissue-derived stem cells under the influence of Fibroblast Growth Factor 2 (FGF2) and other growth and proliferation factors in an animal model. In the presence of FGF2 the observed ADSCs proliferated faster, expressed endothelial surface markers as CD31 or von Willebrand Factor (vWF) and formed tube-like formations in 
matrigel [14]. Following these findings a cross germinal differentiation of adult stem cells with the support of growth of differentiation factors - seems feasible.

There are several similar investigations to support these findings; many other authors come to the conclusion that this cross-germinal specialization cannot be observed reproducibly. Especially the biological functionality of the differentiating cells is questioned critically.

In a clinical context, the capacity of adult stem cells to cross their germinal borderline remains unclear so far; there is particularly no sufficient and finally convincing in-vivo data to support the hypothesis.

Adult stem cells are detected in different tissues:

Mesenchymal stromal or stem cells (MSCs) are fibroblast-like shaped cells and appear ubiquitously in the human organism; they seem to originate from perivascular or adventitial tissue. They have the potential of multilineage differentiation and seem to exert numerous paracrine effects: by secreting different growth and signalling factors they seem to be able to modulate angiogenesis, (anti) inflammation or apoptosis.

The first MSCs described were bone marrow derived cells [15]: they are harvested from bone marrow aspirates. Their cultivation, functional characterization and differentiation behaviour has been investigated since the 1970s. Under standard culture conditions they have to be able to specialize into osteoblasts and chondrocytes.

Adipose derived stromal cells (ADSCs) come with many advantages: their harvest is much easier and safer than the isolation of bone marrow. One is able to produce the cells in larger amounts and the cells can be isolated repeatedly from the same donor region. The isolation and characterization is performed without difficulty and has often been described in literature.

Figure 2 gives a survey of the isolation of hADSCs.

Another interesting source of adult stem cells is the dental pulp stem cells (DPSCs). The probably most attractive feature of these cells that develop from the neural crest is their easy approachability from removed (wisdom) teeth as a by-product of the necessary surgery [16].

Due to their cytological similarity to bone marrow derived stem cells, these dental derived cells are under special investigation concerning not only repair or replacement of dental structures but in musculoskeletal regeneration also. During their characterisation in vitro it could be shown that DPSCs - compared to bone marrow derived cells for - show striking similarity to neuroglia cells and differentiated into neural and endothelial cells reproductively [17].

It has been possible to reprogramme dental pulp cells after third molar removal to induced pluripotent cells (iPS) that differentiated in vitro and in-vivo expressing cell and tissue types from all three germ layers. Even the typical formation of teratoma could be observed [18].

In mouse models of myocardial infarction and hind limb ischemia the therapeutic potential of endothelial and smooth muscle cells derived from DPSC induced pluripotent cells has been demonstrated [19]. These investigations encourage further scientific work with dental pulp derived cells, which are gained in many cases as a by-product of a necessary tooth removal; 


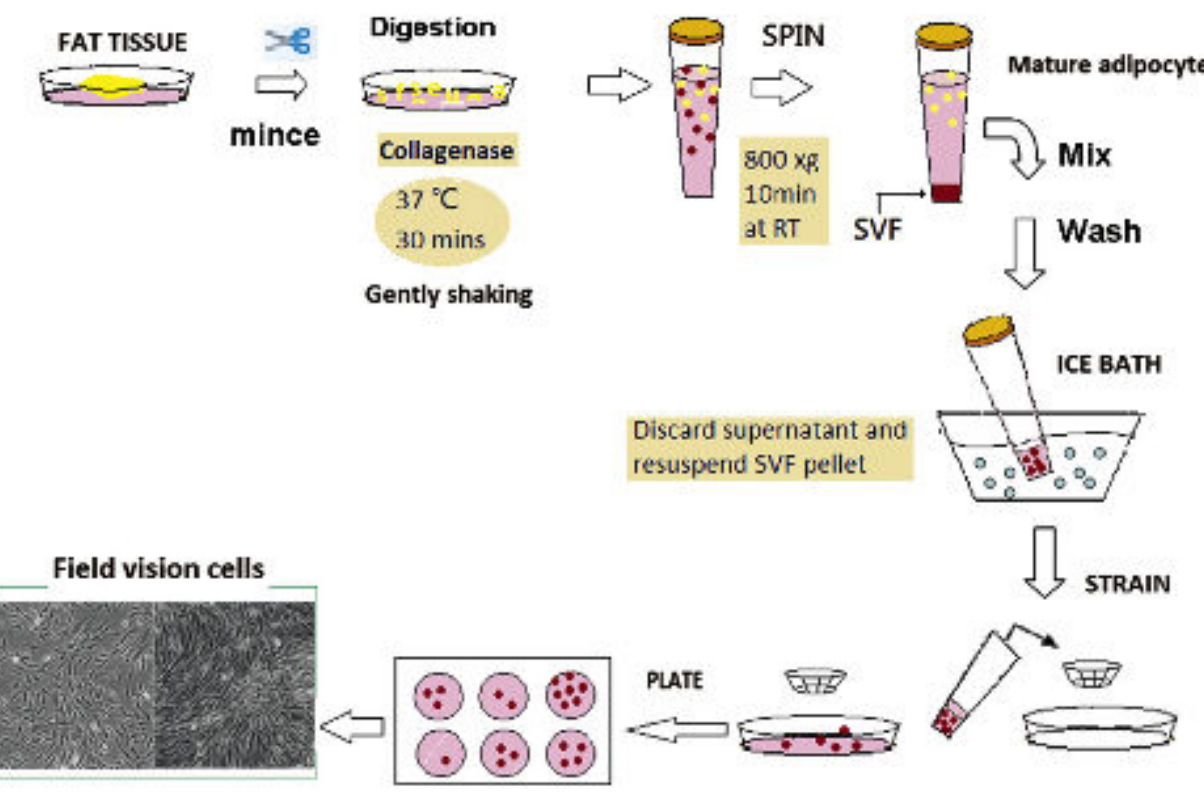

Figure 2. The preparation and isolation process, RT=room temperature, SVF=stromal-vascular cell fraction [3]

in future concepts the dental pulp cells from third molars might be preserved and stored for later use in cell based autologous tissue regeneration.

To combine the good accessibility of adult cells with the excellent differentiating potential another cell source was to be established. Induced pluripotent cells (iPS) surmount the problem of limited access: the cells, for example skin fibroblasts, can be harvested (repeatedly) after informed consent in the mature organism from various tissues as adult cells with differentiating capacity. Therefore they prevail the difficulty of immunoreactivity: they can be harvested autologously. By inducing certain surface/ interaction markers retrovirally, among others Sox2, c-Myc, the so-called Yamanaka factors, these cells forget their initial programming and regress to an earlier precursor stage with enhanced, ES cell-like differentiating properties. Similar to ECS they are capable of unlimited self-renewal in vitro.

The iPS cells show very good proliferative activity; this feature comes with the induction of neoplasia after transplantation, similar to embryonic stem cells.

Their potential and special charm lies in the fact, that with the help of this technique it is generally feasible to isolate an individual cell line from almost every donor. This cell line might be the basis of cell pool, from which many different functional cell and tissue type can be generated autologously for individualized regenerative concepts. In addition iPS cells are used for the construction of disease specific cell and tissue models. With these the potential of therapeutic strategies can be explored.

Figure 3 illustrates the experimental process. 


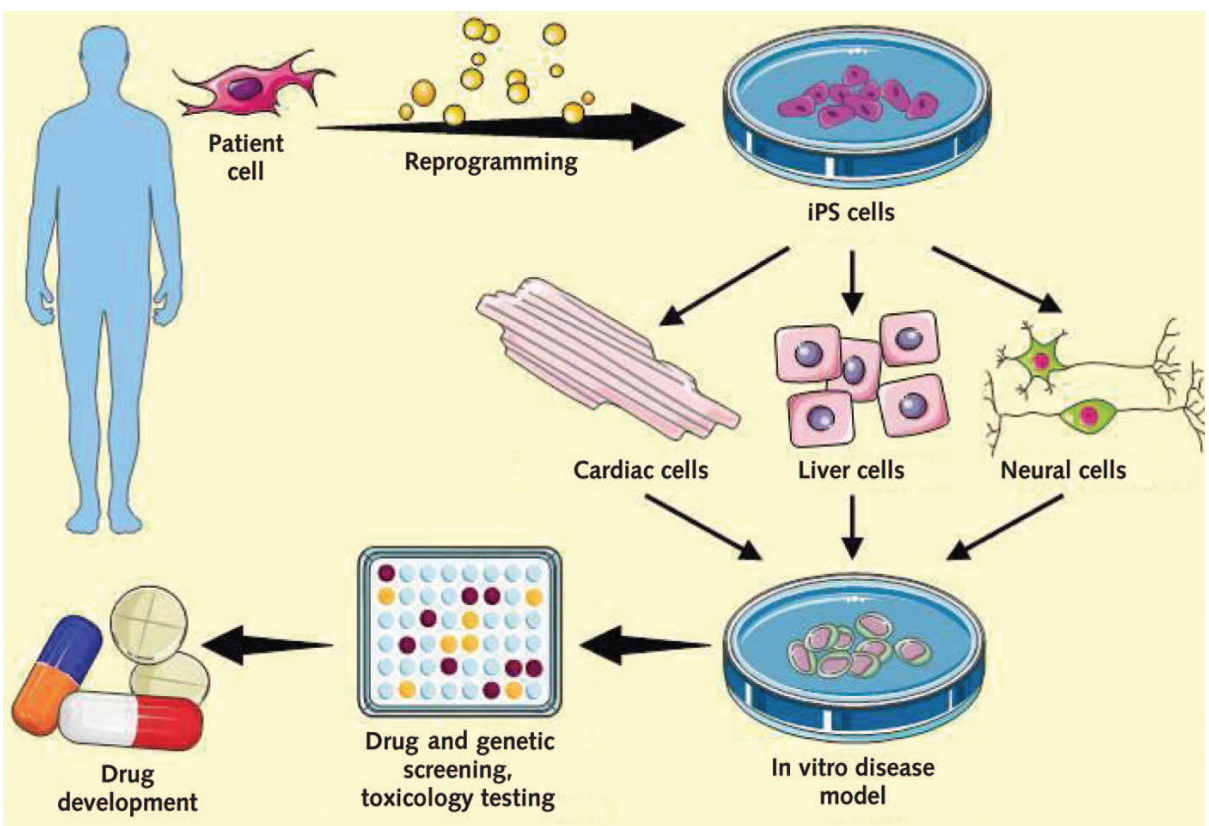

Figure 3. iPS cells as models of different diseases [20, 21]

The harvest, isolation and application of somatic or adult stem cells provide the potential for personalized regenerative concepts. The application especially of autologous cells comes with the advantage of avoiding immunorejection and has verified its prospective in different clinical concepts.

\section{Adipose-derived cells vs. bone marrow-derived cells}

Human adipose derived stromal cells (hADSCs) are localized in different fatty tissues as subcutaneous, visceral and omental fat. Morphologically these cells appear typically as spindle-shaped. Their harvesting and isolation can be easily performed with minimal invasive approach under local anaesthesia with minimal tissue damage and without excessive scarring, for example by liposuction or -aspiration, if necessary the collect can be repeated without difficulty. The cells can be harvested in amounts that up to 500 times higher than mesenchymal stromal cells from other localizations. This feature comes with the advantage that the cells could be implanted at a therapeutic amount without a preparing culturing and expansion phase; thus, adverse culture effects that might impair their biological function and effectiveness can be avoided [22].

In many investigations it was possible to show that the adipose derived cells are able to differentiate within their original embryonic germ layer to chondrocytes or myoblasts. In 
addition some authors state that a capacity for differentiation beyond the cytological restrictions of the mesoderm to neuronal and endothelial cells is possible. Rehman et al. among others described the potential of adipose derived cells for endothelial and macrophage differentiation [23]. These traits make adipose-derived cells an ideal cell source for personalized regenerative concepts including bone or chondral tissue. In addition to that, adipose-derived tissue represents an enormous reservoir of potentially angiogenic cells. Their prospective in therapeutic (neo-)vascularization is not yet fully elucidated.

Table 3 gives a survey of modulating factors that initiate the respective differentiation of ADSCs.

\begin{tabular}{ll}
\hline Adipogenic specialization & $\begin{array}{l}\text { Insulin, IBMX, dexamethasone, rosiglitazone, } \\
\text { indomethacin }\end{array}$ \\
\hline Osteogenic specialization & $\begin{array}{l}1,25 \text {-dihydroxy-cholecalciferol, beta-glycerophosphate, } \\
\text { ascorbic acid }\end{array}$ \\
\hline Neurogenic specialization & EGF, FGF, 5-azacytidine \\
\hline
\end{tabular}

Table 3. Selection of growth factors that support the various differentiations of hADSCs, EGF: epidermal growth factor; FGF: fibroblast growth factor, BMX: 3-isobutyl-1-meyhylxanthine; IGF: insulin-like growth factor, [24]

In in-vitro settings the culture with certain supplements und defined conditions led to the assumption, that a differentiation of ADSCs to hepatocytes of beta cells of the langerhans islets might be feasible, hinting to a probable autologous cell based approach to chronic liver failure or diabetes mellitus. However, these interesting attempts are not ready for therapeutic application yet $[25,26]$.

Bone marrow derived mesenchymal stromal cells (BM-MSCs) were the first pluripotent adult cells to be detected and investigated and therefore are characterized adequately. Bone marrow derived MSCs are gained from bone marrow aspirates, a painful clinical procedure that comes with potential donor site morbidity as an impaired sensitivity of the lateral thigh or pelvis fracture. The output of pluripotent cells is relatively small; only $0.001 \%-0.01 \%$ of the nucleated bone marrow cells can be expected to have differentiating potential, with decreasing percentage in elder donors [22]. BM-MSCs have been applied in various in vitro settings, in animal models and in some clinical trials.

When comparing adipose-and bone marrow derived cells, one has to regard the immunophenotype. The expression of surface markers on both cell types is widely identical. Whereas adipose derived cells tend to express CD34 especially in early stages, bone marrow derived cells and MSCs from other sources do not express this surface marker [27].

Furthermore there is some hint that bone marrow derived MSCs show a significantly reduced proliferation rate compared to other adult pluripotent cells as adipose derived cells or umbilical cord cells [28]. This question is discussed controversially though; harvesting, isolation and culture protocols or conditions as media or various supplements might be responsible for enhanced or reduced proliferation rates - independent from the cell source. 
The question of superior differentiation capacity is debated controversially also. There are many investigations supporting the thesis that the one or the other cell type might be superior in differentiating to an adipogenic or osteogenic direction or they detect no difference at all. In this case, too, the impact of special culture conditions as cause of the different findings has not been ruled out fully.

Finally, the bone marrow and adipose derived cells seem to exert different immunomodulatory effects on their host tissue. In different in vitro studies ADSCs seem to have a more pronounced suppressive effect on the immune response than BM-MSCs. Adipose derived cells were more efficient to inhibit the maturation of dendritic cells and in stimulating the secretion of the immunomodulatory cytokine IL $10[29,30]$.

The analysis of the respective gene expression profile of ADSCs and BM-MSCs indicated that a differential expression of about $13 \%$ of the studied genes differentiates both cell populations on gene level. It was not possible to define certain key markers that clearly identify their origin. Different cell passages or varying culture conditions, too, influence the expression profile [31].

\section{Current concepts of stem cell supported tissue engineering}

The first reported success of the clinical use of autologous differentiating cells in regenerative medicine was the successful treatment of traumatic calvarian defects with a combination of autologous iliac bone, adipose derived stromal cells and fibrin in a degradable scaffold leading to bone formation and good clinical regeneration of the impaired scull [32].

Clinical trials with small patient groups report, that during the treatment of chronic perianal fistula of Crohn patients, a local therapy of the affected area with autologous ADSCs, in combination with fibrin glue, seems to effect a higher healing rate than many other therapeutic strategies [33, 34].

Especially in plastic surgery clinical concepts based on the distinctive properties of adipose derived stem cells are established; actually the cells' potential has been discovered by plastic surgeons [35].

One clinical challenge in plastic surgery is the surgical approach to chronic wounds as diabetic ulcers or skin defects after radiation due to the impaired vascularisation of the affected area. Allogeneic dermis or collagen scaffolds are cultivated with adipose derived pluripotent cells that lead to good clinical results, avoiding the accompanying scarring and donor site morbidity of local flap surgery. The procedure can easily be repeated in larger and deeper defects [36].

Injection of autologous adipose derived stromal cells is effective in the treatment of arterial clotting after filler injection in the face for augmentation purposes. When this complication happens, the area in question becomes sensitive and painful due to the hypoxia of the affected tissue. The arterial obstruction has to be overcome within 4-5 days to avoid tissue necrosis. In case reports it has been described that the injection of autologous ADCs results in reduced ischemia and immediate neo-vascularisation. In Europe the first clinical trial with ADSCs in 
the treatment of cardiovascular diseases as acute myocardial infarction or chronic heart failure have started.

Dental pulp derived stem cells have been studied and applied in regenerative concepts concerning the tooth structures particularly. In an animal model Iohara et al. demonstrated the entire regeneration of the histologically complex pulp tissue including neuro-and vasculogenesis after pulpectomy supported by autologous PDSCs transplantation [37].

According to their original repair and renewal function autologous DPSCs have been eingesetzt to replace dentin. In a porcine model direct pulp capping has been performed using autologous DPSCs together with a synthetic restaurative dental material (beta-tricalcium phosphate); in the follow up control the effective dentin regeneration was demonstrated [38]. The aim to completely replace a missed tooth requires a complex communication, interaction and vice-versa induction of mesenchymal cells and epithelial cells from the oral cavity; different investigative settings aim to orchestrate this complex interaction. Ikeda et al. were able to complete the bioengineering of a primordial tooth that has been inserted in the oral cavity and developed roots and really erupted into the oral cavity [39]. Beyond the dental structures DPSCs have shown their capacity for osteogenic differentiation in vitro as well as in in-vitro trials. Dental pulp derived cells were used among others combined with beta tricalcium phosphate scaffolds in critical size defects in rat calvaria. Annibaldi et al. reported in their preliminary data a trend to increased bone density and mineralisation in the presence of the DPSCs [40].

Beyond their potential concerning hard tissue regeneration, dental pulp derived cells were under investigation in terms of neurological and endocrinological issues also. The hopeful results report an improved and accelerated functional regeneration after focal cerebral ischemia and therapeutic local transplantation of DPSCs; especially the impact of secreted mediators on the repair mechanisms seems to cause the observed effects [41]. Not only a differentiation to hepatocyte-like cells with the ability to store glycogen and to produce urea but also islet-like cells being able to release insulin glucose-dependently has been working out in-vitro and in mice models $[42,43]$.

To date the most encouraging operational area for adult, especially adipose derived stem cells are concepts of bone repair and regeneration. Especially the active physiological behaviour of healthy bone tissue with its permanent remodelling to adapt to mechanical loading or its lack or to accommodate the current nutrient supply makes this tissue an ideal point of application for cell based intervention. Additionally, the therapy of bony critical size defects often comes with clinical problems of tedious distraction, insufficient long-term osteosynthesis or donor site morbidity after autologous bone grafting.

Current research therefore deals with interventional concepts that combine the differentiating precursor cells from adipose tissue with the enormous paracrine effect of these cells, secreting a plethora of proliferation, growth and differentiation factors and thus activating not only the regenerative potential of the resident tissue but also attracting circulating precursor cells.

Structural base of the most regenerative approaches is still a 3D scaffold to support the homing of the proliferating cells. Not only are the mechanical properties of these scaffolds of impor- 
tance, their composition affects the biology of the cells also: Marino et al. were able to depict, that the osteogenic differentiation of human adipose derived stem cells onto a porous betatricalcium phosphate (beta-TCP) matrix was triggered by the scaffold alone without the use of differentiative media [44]. This effect might be enhanced by modern composite scaffolds: akermanite scaffolds, consisting of calcium-, magnesium-, and silicon-containing ceramic show improved stability and better degradation. In addition they seem to positively influence the functionality of differentiating cells by inducing their calcium deposition $[45,46]$.

\section{Future prospect}

In different experimental settings the transplanted pluripotent cells do not exert their regenerative potential by actually differentiating and replacing damages tissue but exert their effects by secreting growth, proliferation and differentiation factors and thus in a paracrine way or by cell-cell interaction.

In terms of stem cell science many biological and technical questions remain unanswered so far and years of intense research lay ahead before autologous pluripotent cells become a standard tool in clinical routine.

The current restrictions of embryonic stem cell research are necessary to avoid abuse but the promising, steadily growing comprehension about adult stem cells and induced pluripotent cells with their ability to regain their original differentiating potential seem to hint to the fact that these cells might be the target of interest in future regenerative concepts in personalized medicine.

The challenge of the coming decade of stem cell research will have to deal with safety issues, the problem to reliably avoid oncogenic growth and to develop reproducible, secure and payable concepts of cell isolation, culturing, (re-) programming and storage. There have to be protocols for the autologous transplantation to the region of interest and techniques to improve cell homing.

Despite many unanswered questions the future of many regenerative concepts will be supported by the potential of differentiating cells.

\section{Author details}

Susanne Jung ${ }^{1,2^{*}}$ and Johannes Kleinheinz $\mathrm{z}^{1,2}$

*Address all correspondence to: Susanne.Jung@ukmuenster.de

1 Department of Cranio-Maxillofacial Surgery, University Hospital Muenster, Germany

2 Research Unit Vascular Biology of Oral Structures (VABOS), Germany 


\section{References}

[1] Mountford JC. Human embryonic stem cells: origins, characteristics and potential for regenerative therapy, Transfus Med. 2008;18(1) 1-12.

[2] Eggan K, Jaenisch R. Differentiation of F1 embryonic stem cells into viable male and female mice by tetraploid embryo complementation, Methods Enzymol 2003;365 2539.

[3] Cheng H. Human adipose-derived stem cells: Isolation, characterization and current application in regeneration medicine. Genomiv Medicine, Biomarkers and Health Sciences. 2011;3 53-62.

[4] Jarocha D, Lukasiewich E, Majka M. Advantage of mesenchymal stem cells (MSC) expansion directly from purifiedbone marrow CD105+and CD271+cells,Folia Histochem Cytobiol 2008;46 307-314.

[5] Bianco P, Robey PG, Simmons PJ. Mesenchymal stem cells: revisiting history, concepts and assays. Cell Stem Cell. 2008;2(4) 313-9.

[6] Bai X, Yan Y, Song YH, Seidensticker M, Rabinovich B, Metzele R, Bankson JA, Vykoukal D, Alt E. Both cultured and freshly isolated adipose tissue-derived stem cells enhance cardiac function after acute myocardial infarction. Eur Heart J. 2010;31(4) 489-501.

[7] Tzameret A, Sher I, Belkin M, Treves AJ, Meir A, Nagler A, Levkovitch-Verbin H, Barshack I, Rosner M, Rotenstreich Y. Transplantation of human bone marrow mesenchymal stem cells as a thin subretinal layer ameliorates retinal degeneration in a rat model of retinal dystrophy, Exp Eye Res. 2014;118 135-44. doi: 10.1016/j.exer. 2013.10.023.

[8] Horwitz EM, Gordon PL, Koo WK, Marx JC, Neel MD, McNall RY, Muul L, Hofmann $\mathrm{T}$. Isolated allogeneic bone marrow-derived mesenchymal cells engraft and stimulate growth in children with osteogenesis imperfecta: Implications for cell therapy of bone, Proc Natl Acad Sci (U S A). 2002;99 8932-8937.

[9] Ortiz LA, Gambelli F, McBride C, Gaupp D, Baddoo M, Kaminski N, Phinney DG. Mesenchymal stem cell engraftment in lung is enhanced in response to bleomycin exposure and ameliorates its fibrotic effects, Proc Natl Acad Sci (U S A) 2003;100 84078411.

[10] Phinney DG, Isakova I. Plasticity and therapeutic potential of mesenchymal stem cells in the nervous system, Curr Pharm Des 2005;11 1255-1265.

[11] Stojkovic M, Lako M, Strachan T et al. Derivation, growth and applications of human embryonic stem cells, Reproduction. 2004;128 259-67. 
[12] Przyborski SA. Differentiation of human embryonic stem cells after transplantation in immune-deficient mice, Stem Cells. 2005;23(9) 1242-1250.

[13] Krampera M, Marconi S, Pasini A, Galie M, Rigotti G, Mosna F, Tinelli M, Lovato L, Anghileri $\mathrm{E}$ et al. Induction of neural-like differentiation in human mesenchymal stem cells derived from bone marrow, fat, spleen and thymus, Bone 2007;40 382-490.

[14] Ning H, Liu G, Lin G, Yang R, Lue TF, Lin CS. Fibroblast growth factor 2 promotes endothelial differentiation of adipose tissue-derived stem cells, J Sex Med. 2009;6(4) 967-79.

[15] Friedenstein AJ, Petrakova KV, Kurolesova AI, Frolova GP. Heterotopic of bone marrow. Analysis of precursor cells for osteogenic and hematopoietic tissues, Transplantation 1968;6 230-247.

[16] Gronthos S, Mankani M, Brahim J et al. Postnatal human dental pulp stem cells (DPSCs) in vitro and in vivo, Proc Natl Acad Sci 2000;97 13625-13630.

[17] Karaoz E, Demircan PC, Saglam Ö et al. Human dental pulp stem cells demonstrate better neural and epithelial stem cell properties than bone marrow-derived mesenchymal stem cells, Histochem Cell Biol 2011;136 455-473.

[18] Yan X, Qin H, Qu C et al. iPS cells reprogrammed from human mesenchymal-like stem/progenitor cells of dental tissue origin, Stem Cells Dev 2010;19 469-480.

[19] Yoo CH, Na HJ, Lee DS et al. Endothelial progenitor cells from human dental pulpderived iPS cells as a therapeutic target for ischemic vascular diseases, Biomaterials 2013;34 8149-8160.

[20] Power C, Rasko JE. Promises and challenges of stem cell research for regenerative medicine, Ann Intern Med. 2011;155(10) 706-713.

[21] Fong CY, Gauthaman K, Bongso A. Teratomas from pluripotent stem cells: A clinical hurdle, J Cell Biochem. 2010;111(4) 769-781.

[22] Bernardo ME, Locatelli F, Fibbe WE. Mesenchymal stromal cells. Ann N Y Acad Sci. 2009;1176 101-17.

[23] Rehman J, Traktuev D, Li J, et al. Secretion of angiogenic and antiapoptotic factors by human adipose stromal cells, Circulation. 2004;109 1292e1298.

[24] Huang SJ, Fu RH, Shyu WC, Liu SP, Jong GP, Chiu YW, Wu HS, Tsou YA, Cheng CW, Lin SZ. Adipose-derived stem cells: isolation, characterization, and differentiation potential, Cell Transplant.2013;22(4) 701-9.

[25] Lue J, Lin G, Ning H, et al. Transdifferentiation of adipose-derived stem cells into hepatocytes: a new approach, Liver Int. 2010;30 913e922. 
[26] Kajiyama H, Hamazaki TS, Tokuhara M, et al. Pdx1-transfected adipose tissue-derived stem cells differentiate into insulin-producing cells in vivo and reduce hyperglycemia in diabetic mice, Int J Dev Biol. 2010;54 699e705.

[27] Gronthos S, DM Franklin, HA Leddy, PG Robey, RW Storms and JM Gimble. Surface protein characterization of human adipose tissue-derived stromal cells, J Cell Physiol 2001;189 54-63.

[28] Kern S, Eichler H, Stoeve J, Kluter K, Bieback K. Comparative analysis of mesenchymal stem cells from bone marrow, umbilical cord blood, or adipose tissue, Stem Cells 2006;24 1294-1301.

[29] Noel D, Caton D, Roche S, Bony C, Lehmann S, Casteilla L, Jorgensen C, Cousin B. Cell specific differences between human adipose-derived and mesenchymal-stromal cells despite similar differentiation potentials, Exp Cell Res 2008;314 1575-1584.

[30] Ivanova-Todorova E, Bochev I, Mourdjeva M, Dimitrov R, Bukarev D, Kyurkchiev S, Tivchev P, Altunkova I, Kyurkchiev DS. Adipose tissue-derived mesenchymal stem cells are more potent suppressors of dendritic cells differentiation compared to bone marrow-derived mesenchymal stem cells, Immunol Lett 2009;126 37-42.

[31] Strioga M, Viswanathan S, Darinskas A, Slaby O, Michalek J. Same or Not the Same? Comparison of Adipose Tissue-Derived Versus Bone Marrow-Derived Mesenchymal Stem and Stromal Cells, Stem Cells Dev. 2012;21(14) 2724-52.

[32] Lendeckel S, Jodicke A, Christophis P, et al. Autologous stem cells (adipose) and fibrin glue used to treat widespread traumatic calvarial defects: case report, J Craniomaxillofac Surg. 2004;32 370e373.

[33] Garcia-Olmo D, Garcia-Arranz M, Herreros D, et al. A phase I clinical trial of the treatment of Crohn's fistula by adipose mesenchymal stem cell transplantation, Dis Colon Rectum. 2005;48 1416e1423.

[34] Garcia-Olmo D, Herreros D, Pascual I, et al. Expanded adipose-derived stem cells for the treatment of complex perianal fistula: a phase II clinical trial, Dis Colon Rectum. $2009 ; 5279 \mathrm{e} 86$.

[35] Zuk PA, Zhu M, Mizuno H, Huang J, Futrell JW, Katz AJ, Benhaim P, Lorenz HP, Hedrick MH. Multilineage cells from human adipose tissue: implications for cellbased therapies, Tissue Eng. 2001;7 211-28.

[36] Yun MJ, Park JU, Kwon ST. Surgical options for malignant skin tumors of the hand, Arch Plast Surg. 2013;40 238-43.

[37] Iohara K, Zheng L, Ito M, Ishizaka R, Nakamura H, Into T, Matsushita K, Nakashima M. Regeneration of dental pulp after pulpotomy by transplantation of CD31(-)/ CD146(-) side population cells from a canine tooth, Regen Med. 2009;4(3) 377-85. 
[38] Zheng Y, Wang XY, Wang YM et al. Dentin regeneration using deciduous pulp stem/ progenitor cells, J Dent Res 2012;91 676-682.

[39] Ikeda E, Morita R, Nakao K et al. Fully functional bioengineered tooth replacement as an organ replacement therapy, Proc Natl Acad Sci U S A 2009;106 13475-13480.

[40] Annibali S, Bellavia D, Ottolenghi L, Cicconetti A, Cristalli MP, Quaranta R, Pilloni A. Micro-CT and PET analysis of bone regeneration induced by biodegradable scaffolds as carriers for dental pulp stem cells in a rat model of calvarial "critical size" defect: Preliminary data. J Biomed Mater Res B Appl Biomater. 2014;102(4) 815-25.

[41] Leong WK, Henshall TL, Arthur A, et al. Human adult dental pulp stem cells enhance poststroke functional recovery through non-neural peplacement mechanisms, Stem Cells Transl Med 2012;1 177-187.

[42] Patil R, Kumar BM, Lee WJ et al. Multilineage potential and proteomic profiling of human dental stem cells derived from a single donor, Exp Cell Res 2013;20 92-107.

[43] Kanafi MM, Rajeshwari YB, Gupta S et al. Transplantation of islet-like cell clusters derived from human dental pulp stem cells restores normoglycemia in diabetic mice, Cytotherapy 2013;15 1228-1236.

[44] Marino G, Rosso F, Cafiero G, Tortora C, Moraci M, Barbarisi M, Barbarisi A. Betatricalcium phosphate 3D scaffold promote alone osteogenic differentiation of human adipose stem cells: in vitro study, J Mater Sci Mater Med. 2010;21(1) 353-63.

[45] Barba M, Cicione C, Bernardini C, Michetti F, Lattanzi W. Adipose-derived mesenchymal cells for bone regereneration: state of the art. Biomed Res Int. 2013;2013 416391.

[46] Zanetti A, McCandless G, Chan J, Gimble J, Hayes D. Characterization of novel akermanite:poly- $\varepsilon$-caprolactone scaffolds for human adipose-derived stem cells bone tissue engineering, J Tissue Eng Regen Med. 2012 doi: 10.1002/term.1646. 